\title{
ON CRACKS AND DISLOCATIONS IN SHAFTS UNDER TORSION*
}

\section{By ALEXANDER WEINSTEIN (University of Maryland)}

1. Introduction. Several decades ago W. Arndt [1] showed that the theory of shafts of revolution under torsion can be reduced to the investigation of axially symmetric motions of a fictitious incompressible fluid in a space of five dimensions. Let $(x, y)$ be the meridian plane of the shaft, $x$ being the axis of symmetry. All relevant quantities are determined by the potential $\Phi(x, y)$ and the stream function $\Psi(x, y)$ which satisfy the generalized Stokes-Beltrami equations

$$
y^{3} \Phi_{x}=\Psi_{y}, \quad y^{3} \Phi_{y}=-\Psi_{x} .
$$

The displacement of a particle is perpendicular to the meridian plane and is given by the formula $v=y \Phi(x, y)$. The stresses in the meridian plane are $\tau_{\theta x}=y \Phi_{x}$ and $\tau_{\theta y}=y \Phi_{y}$, where $\theta$ denotes the polar angle in the plane perpendicular to the $x$-axis. ${ }^{* *}$ Any streamline $\Psi=$ const. is free from stress and can be taken as the profile of a free surface bounding the shaft.

By analogy with ordinary hydrodynamics, Arndt developed the method of sources and sinks in a space of five dimensions using however source distribution only on the axis of symmetry. Recently the method of sources and sinks has been extended by the present author to distributions outside of the axis of symmetry [2], [3]. This work was extended by A. Van Tuyl [4] and by M. A. Sadowsky and E. Sternberg [5]. The present paper gives new application of this theory to problems of cracks and dislocations in a shaft under torsion. A new and simple correspondence principle will be used connecting the method of sources and sinks with electrostatic problems in Generalized Axially Symmetric Potential Theory [6], [7] which will be denoted by the abbreviation GASPT.

2. The correspondence principle. GASPT deals with equations of the form

$$
y^{p} \varphi_{x}(x, y)=\psi_{y}(x, y), \quad y^{p} \varphi_{y}(x, y)=-\psi_{x}(x, y)
$$

for all non-negative values of the parameter $p$. For $p=1,2,3, \cdots \varphi$ represents an axially symmetric potential in the meridian plane of a space of $p+2$ dimensions. The case $p=0$ corresponds to harmonic functions in the plane. In all cases $\psi$ is the corresponding stream function. For clarity's sake we shall sometimes write $\varphi\{p\}$ and $\psi\{p\}$ in place of $\varphi$ and $\psi$. We have from (2.1) the following equations

$$
\frac{\partial}{\partial x}\left(y^{p} \frac{\partial \varphi}{\partial x}\right)+\frac{\partial}{\partial y}\left(y^{p} \frac{\partial \varphi}{\partial y}\right)=0, \quad \frac{\partial}{\partial x}\left(y^{-p} \frac{\partial \psi}{\partial x}\right)+\frac{\partial}{\partial y}\left(y^{-p} \frac{\partial \psi}{\partial y}\right)=0 .
$$

We see that $\varphi$ and $\psi$ satisfy the same equation only in the case $p=0$, which corresponds to the well known fact that in plane hydrodynamics a stream function can be used as a velocity potential and vice versa. For $p \geqq 0$, we use a fundamental identity [6, p. 351, (48)], which can be written as follows

$$
\psi\{p\}=C y^{p+1} \varphi\{p+2\},
$$

where $C$ is an arbitrary constant. This identity permits us to obtain from a stream function $\psi\{p\}$ a certain potential $\varphi\{p+2\}$ and vice versa.

${ }^{*}$ Received June 7, 1951. This work has been done under the sponsorship of the Office of Naval Research.

${ }^{* *}$ We have put Lamé's constant $\mu$ equal to unity. 
3. The method of sources and sinks. In the case of shafts, as well as in any other cases, solutions of certain problems are obtained by putting, for a constant $U$,

$$
\Phi\{p\}=U x-\varphi\{p\}, \quad \Psi\{p\}=U(p+1)^{-1} y^{p+1}-\psi\{p\} .
$$

In the usual cases $\varphi$ and $\psi$ are due to sources distributed in a finite domain. For the theory of shafts, $p$ is to be taken equal to 3 . Our main problem is to determine the lines $\Psi\{p\}=$ constant, the line $\Psi\{p\}=0$ being the line enclosing the singularities. We assume in the following that this line is closed. By (2.3), we may put $\psi\{p\}=$ $U(p+1)^{-1} y^{p+1} \varphi\{p+2\}$. Using the notation $\varphi\{p+2\}=\varphi^{*}(x, y)$, we have (3.1)

$$
\Psi\{p\}=U(p+1)^{-1} y^{p+1}\left(1-\varphi^{*}(x, y)\right) .
$$

This shows that to the closed streamline $\Psi\{p\}=0$ correspond the level line $\varphi^{*}=$ $\varphi\{p+2\}=1$. As $\psi\{p\}$ tends to $U(p+1)^{-1} y^{p+1}$ at infinity, we see from (3.2) that $\varphi\{p+2\}$ must tend to zero for $x^{2}+y^{2} \rightarrow \infty$. In this way the problem for $\Psi\{p\}$ is now reduced by our correspondence principle to the electrostatic problem for $\varphi\{p+2\}$.

4. The crack problem. A flat interior circular crack perpendicular to the axis of symmetry of the shaft is defined as a circular disc separating the material. It is assumed that no stress is acting across the surface of the disc. The definition of the crack adopted here is the same as that used in another problem by M. A. Sadowsky and E. Sternberg [8]. Let us note that A. E. Green [9] uses the word crack in a different sense, namely in order to denote the displacement on the surface of a body due to localized pressure.

Let $x=0, y=0$ be the center of our disc of radius $b$. By Arndt's theory, the boundary condition is given by $\Psi\{3\}=0$ on both sides of the segment $x=0,0 \leqq y \leqq b$ in the upper half of the meridian plane, $\Psi$ being an even function of $x$. The problem is, by (3.2), reduced to the determination of the electrostatic potential $\varphi\{5\}$ of a disc of radius $b$ in a space of seven dimensions. This problem will be now solved by using ellipsoidal coordinates. Let

$$
\frac{x^{2}}{\lambda}+\frac{y^{2}}{\lambda+b^{2}}=1
$$

be a family of confocal ellipses which generate through a rotation about the $x$-axis a family of oblate spheroids $\lambda=$ constant in a space of seven dimensions. The value $\lambda=0$ corresponds to the disc. Let us put

$$
\varphi^{*}=\frac{8 b^{5}}{3 \pi} \int_{\lambda}^{\infty} \frac{d u}{\left(u+b^{2}\right)^{3}(u)^{1 / 2}}
$$

Then $\varphi^{*}$ satisfies (2.2) for $p=5$, vanishes at infinity and takes the constant value one on the disc. The integral in (4.2) can be expressed in terms of elementary functions, the result being the formula

$$
\varphi^{*}=\frac{2}{\pi} \operatorname{arccot} \frac{(\lambda)^{1 / 2}}{b}-\frac{2 b}{3 \pi} \frac{\left(3 \lambda+5 b^{2}\right)(\lambda)^{1 / 2}}{\left(\lambda+b^{2}\right)^{2}} .
$$

By Sec. 3 we have

$$
\psi\{3\}=4^{-1} U y^{4} \varphi^{*}
$$

In order to obtain $\varphi\{3\}$ we introduce in the $x, y$-plane elliptic coordinates by the formulae

$$
x=b \sinh \xi \cos \eta, \quad y=b \cosh \xi \sin \eta .
$$


By comparison with (4.1), see that

$$
b^{2} \sinh ^{2} \xi=\lambda, \quad b^{2} \cosh ^{2} \xi=\lambda+b^{2}
$$

As (4.5) defines a conformal transformation, i.e. a local rotation and uniform dilation of the coordinate system, we have, in place of (2.1)

$$
y^{p} \varphi_{\xi}=\psi_{\eta}, \quad y^{p} \varphi_{\eta}=-\psi_{\xi},
$$

where by (4.5), $y=b \cosh \xi \sin \eta$.

By (4.3) and (4.4) we have

$$
\psi\{3\}=U b^{4}(6 \pi)^{-1}\left\{3 \cosh ^{4} \xi \operatorname{arccot}(\sinh \xi)-3 \sinh ^{3} \xi-5 \sinh \xi\right\} \sin ^{4} \eta .
$$

To find $\varphi$, we use (4.6) for $p=3$, which yields $\varphi_{\eta}$. By an integration we obtain finally

$$
\varphi\{3\}=U b \pi^{-1}\left\{\frac{2}{3 \cosh ^{2} \xi}+2 \sinh \xi \operatorname{arccot}(\sinh \xi)-2\right\} \cos \eta .
$$

Obviously $\varphi$ vanishes for $x+y^{2} \rightarrow \infty$.

The formulae (4.8) and (4.7) together with (3.1), taken for $p=3$ give the solution of the crack problem in elliptic coordinates. The same solution can be also expressed in terms of $x$ and $y$. In fact, the quantity

$$
s(x, y)=\left[x^{2}+|y-b|^{2}\right]^{1 / 2}+\left[x^{2}+|y+b|^{2}\right]^{1 / 2}
$$

is a function $f(\lambda)$ of $\lambda$ alone. Putting $y=0$ in (4.1) we find $\lambda=x^{2}$. Therefore on the ellipse $\lambda=$ constant we have $s=s(x, 0)=2\left(x^{2}+b^{2}\right)^{1 / 2}=2\left(\lambda+b^{2}\right)^{1 / 2}$. This equation yields

$$
\lambda=\frac{1}{4} s^{2}(x, y)-b^{2} .
$$

This value of $\lambda$ can be substituted in (4.6), (4.7), and (4.8) and gives us explicit formulas for $\varphi(x, y)$ and $\psi(x, y)$ in terms of $x$ and $y$.

The same functions $\varphi$ and $\psi$ can also be expressed in terms of integrals involving products of Bessel functions. However, such integrals are less suitable for the discussion. In fact, these new expressions should be rather considered as new formulae yielding certain integrals in terms of elementary functions. The corresponding results (also for an arbitrary value of the parameter $p$ ) will be published elsewhere.

5. Discussion of the results in the crack problem. For the discussion, we have to use the formulae for displacements and stress given in the Introduction, and the formulas for $\Phi, \Psi, \varphi$ and $\psi$ given in paragraphs 3 and 4 . It will suffice to note the following results. The transverse displacement $v(x, y)$ is given for $x=0$ by the formulae

$$
v(0, y)=\left\{\begin{array}{cll} 
\pm \frac{4}{3 \pi} U y\left(b^{2}-y^{2}\right)^{1 / 2}, & \text { for } & 0 \leqq y \leqq b, \\
0, & \text { for } & y>b .
\end{array}\right.
$$

The signs + and - correspond here and in the following to the positive and to the negative side of the $y$-axis respectively. The stress $\tau_{\theta x}$ is zero on the disc, i.e. for $x=0$, $0 \leqq y \leqq b$ but becomes infinite like $(y-b)^{-1 / 2}$ for $y>b$. The stress $\tau_{\theta y}$ tends to infinity in the same manner for $x=0, y \nearrow b$. For large values of $x^{2}+y^{2}, \varphi^{*}$ tends to zero like $\left(x^{2}+y^{2}\right)^{-5 / 2}$. Therefore the formula $\Psi=4^{-1} U y^{4}\left(1-\varphi^{*}\right)$ shows that already for 
moderate values of $y$, a streamline $\Psi=$ constant is essentially parallel to the axis of symmetry, so that we have a nearly cylindrical beam with a circular crack.

It is clear that the method presented here can be immediately applied to cavities of the form of oblate spheroids.

6. Dislocations. According to the classical theory of Volterra a surface of dislocation is a surface of discontinuity for the displacements. The stresses remain continuous across this surface and the displacements on both sides of a dislocation differ only by a rigid body motion. In the case of a shaft under torsion we take as the surface of the dislocation the same circular disc as in the crack problem. The only admissable dislocation is, in this case, a rigid rotation of the negative side of the disc relative to its positive side. Such a dislocation is readily obtained by taking in (3.1) for $\varphi=\varphi\{3\}$ the potential of a uniform magnetic shell in a five dimensional space. By a specialization of a general formula $[7$, p. $76,(6.1)]$ we have

$$
\varphi=M b^{2} y^{-1} \int_{0}^{\infty} e^{-x t} J_{1}(y t) J_{2}(b t) d t, \quad x \geqq 0
$$

and

$$
\psi=-M b^{2} y^{2} \int_{0}^{\infty} e^{-x t} J_{2}(y t) J_{2}(b t) d t, \quad x \geqq 0 .
$$

For $x \leqq 0$, we have $\varphi(-x, y)=-\varphi(x, y), \psi(x, y)=\psi(-x, y)$. In (6.1) and (6.2) $M$ is a positive constant and the $J_{q}$ denote Bessel functions. In view of the results given in [7] we have the following values for the displacement $v$ in the plane of the disc:

$$
v(0, y)=\left\{\begin{array}{ccc} 
\pm M y, & \text { for } & y<b \\
0, & \text { for } & y>b
\end{array} .\right.
$$

Moreover we have

$$
\lim v(x, y)=M b(\pi-\beta) \pi^{-1}
$$

for $x \rightarrow 0, y \rightarrow b$ and $(b-y) x^{-1} \rightarrow \cot \beta$, where $x \geqq 0$ and $0 \leqq \beta \leqq \pi$. By a well known property of uniform magnetic shells which remain valid in any number of dimensions, the stresses $\tau_{\theta x}$ and $\tau_{\theta y}$ remain continuous across the disc. However, they become infinite in the neighborhood of its rim like $(y-b)\left[x^{2}+(b-y)^{2}\right]^{-1}$ and $x\left[x^{2}+(b-y)^{2}\right]^{-1}$ respectively. The connection of the formulae (6.1) and (6.2) with elliptic integrals analogous to those given in [4] and [5] will be given elsewhere. Further applications of "generalized electrostatics" are given in [10].

The author wishes to thank Dr. H. F. Weinberger for valuable discussion on the material of this paper.

\section{References}

1. W. Arndt, Die Torsion von Wellen, Thesis, Goettingen, 1916.

2. A. Weinstein, On axially symmetric flows, Q. Appl. Math., 5, 429-444 (1948).

3. A. Weinstein, On the torsion of shafts of revolution, Proc. 7th Internat. Congr. Appl. Mech., Vol. 1, 1948, pp. 108-119.

4. A. Van Tuyl, On the axially symmetric flow around a new family of halfbodies, Q. Appl. Math., 7, 399409 (1950). 
5. M. A. Sadowsky and E. Sternberg, Elliptical integral representation of axially symmetric flows, Q. Appl. Math., 8, 113-126 (1950).

6. A. Weinstein, Discontinuous integrals and generalized potential theory, Transact. Amer. Math. Soc., 63, 342-354 (1948).

7. A. Weinstein, Transonic flow and generalized axially symmetric potential theory, Proc. Aeroball. Res. Symposia, Naval Ordnance Laboratory, 1949, pp. 73-82.

8. M. A. Sadowsky and E. Sternberg, Stress concentration around a triaxial ellipsoidal cavity, J. Appl. Mech., 71, 149-159 (1949).

9. A. E. Green, On Boussinesq's problem and penny-shaped cracks, Cambridge Phil. Soc., 45, 251-256 (1948) (this paper contains references to previous work by J. W. Harding and I. N. Sneddon).

10. A. Weinstein, The method of singularities in the physical and in the hodograph plane, to appear in the Proc. Symposia Appl. Math., vol. 4, McGraw-Hill Book Company, Inc., New York, 1952.

\section{INDUCED MASS WITH FREE BOUNDARIES*}

\section{BY GARRETT BIRKHOFF (Harvard University)}

The concept of induced mass, and some of its properties, are extended to the case of an incompressible liquid having a free surface. The usual ${ }^{1}$ treatment of the case of a non-viscous fluid extending to infinity must be considerably changed to do this.

1. Minimum principle. Let $R$ be a region filled with an incompressible liquid, bounded in part by a wetted wall $W$, and in part by a free surface $S$ at constant pressure $p_{0}$. The region $R$ moves with the liquid, and may extend to infinity in some directions.

Suppose the fluid accelerated from rest, by an acceleration of $W$ whose inward normal component toward the liquid is an arbitrary function $f(\mathbf{x})$ of position. Letting $\mathfrak{u}=$ $\mathbf{u}(\mathbf{x} ; t)$ denote liquid velocity and $\mathbf{a}=\partial \mathbf{u} / \partial t$ denote acceleration, clearly

$$
\operatorname{Div} \mathbf{a}=\Sigma \partial\left(\partial u_{k} / \partial t\right) / \partial x_{k}=\partial\left(\Sigma \partial u_{k} / \partial x_{k}\right) / \partial t=0,
$$

by incompressibility. Similarly, the Navier-Stokes equations with gravity neglected are ${ }^{2}$

$$
D u_{i} / D t=-\partial p / \rho \partial x_{i}+\nu \nabla^{2} u_{i} .
$$

Since $\mathfrak{u}=0$ initially, the initial acceleration therefore satisfies $a_{i}=D u_{i} / D t=-\partial p / \rho \partial x_{i}$, or, setting $A=\left(p_{0}-p\right) / \rho$,

$$
\mathbf{a}=\nabla A, \quad \text { where } \quad p=p_{0}-\rho A, \quad \text { initially. }
$$

Combining (3) with (1), we get

$$
\nabla^{2} A=0 \quad \text { in } R .
$$

The free surface condition is simply

$$
A=0 \quad \text { on } \quad S .
$$

Finally, by continuity, we have

$$
a_{\text {normal }}=\partial A / \partial n=f(\mathbf{x}) \quad \text { on } W .
$$

*Received July 16, 1951.

'Given in [1], Chap. V; in [3]; and in Chap. VI of Lamb's Hydrodynamics.

${ }^{2}$ Here and below $D / D t$ is the substantial derivative $\partial / \partial t+u_{k} \partial / \partial x_{k}$, while $\partial / \partial n$ denotes the inward normal derivative on the surface of the liquid. 